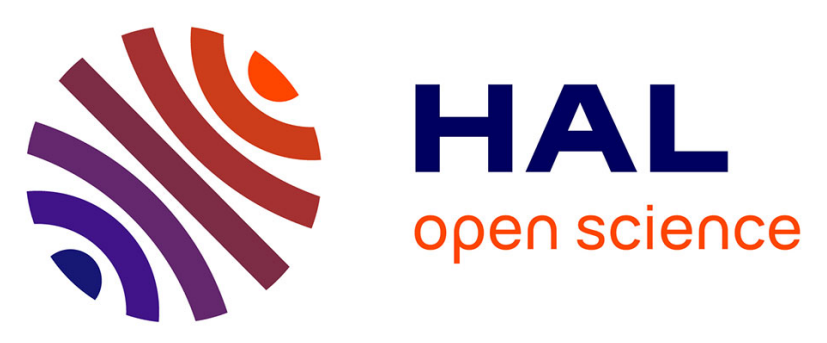

\title{
Exploration of biomedical dendrimer space based on in-vitro physicochemical parameters: key factor analysis (Part 1)
}

Serge Mignani, João Rodrigues, Rene Roy, Xiangyang Shi, Valentin Ceña, Said El Kazzouli, Jean Pierre Majoral

\section{- To cite this version:}

Serge Mignani, João Rodrigues, Rene Roy, Xiangyang Shi, Valentin Ceña, et al.. Exploration of biomedical dendrimer space based on in-vitro physicochemical parameters: key factor analysis (Part 1). Drug Discovery Today Biosilico, 2019, 24 (5), pp.1176-1183. 10.1016/j.drudis.2019.02.014 . hal02334985

\section{HAL Id: hal-02334985 \\ https://hal.science/hal-02334985}

Submitted on 22 Oct 2021

HAL is a multi-disciplinary open access archive for the deposit and dissemination of scientific research documents, whether they are published or not. The documents may come from teaching and research institutions in France or abroad, or from public or private research centers.
L'archive ouverte pluridisciplinaire HAL, est destinée au dépôt et à la diffusion de documents scientifiques de niveau recherche, publiés ou non, émanant des établissements d'enseignement et de recherche français ou étrangers, des laboratoires publics ou privés.

\section{(ㄷ)(1) $\$$}

Distributed under a Creative Commons Attribution - NonCommerciall 4.0 International 


\title{
Exploration of biomedical dendrimer space based on in- vitro physicochemical parameters: key factor analysis
}

\author{
Serge Mignani, ${ }^{1,2,3,4}$ João Rodrigues, ${ }^{3,5}$, René Roy, ${ }^{3}$ Xiangyang Shi, ${ }^{6}$ Valentin \\ Ceña, ${ }^{7,8}$ Saïd El Kazzouli, ${ }^{9}$ and Jean-Pierre Majoral ${ }^{1,10,11}$
}

${ }^{1}$ China-France Joint Laboratory for Healthcare Theranostics, Shanghai Tenth People's Hospital, Tongji University School of Medicine, Shanghai, China

${ }^{2}$ Université Paris Descartes, PRES Sorbonne Paris Cité, CNRS UMR 860, Laboratoire de Chimie et de Biochimie Pharmacologiques et Toxicologique, 45, rue des Saints Peres, 75006 Paris, France

${ }^{3}$ CQM - Centro de Química da Madeira, MMRG, Universidade da Madeira, Campus da Penteada, 9020-105 Funchal, Portugal

${ }^{4}$ Glycovax Pharma, 424 Guy Street, Suite 202, Montréal, QC, H3J 1S6, Canada

${ }^{5}$ School of Materials Science and Engineering/Center for Nano Energy Materials, Northwestern Polytechnical University, Xi'an 710072, China

${ }^{6}$ State Key Laboratory for Modification of Chemical Fibers and Polymer Materials, College of Chemistry, Chemical Engineering and Biotechnology, Donghua University, Shanghai 201620, China

${ }^{7}$ Unidad Asociada Neurodeath, Universidad de Castilla-La Mancha, 02006 Albacete, Spain

${ }^{8}$ Centro de Investigación Biomédica en Red para Enfermedades Neurodegenerativas, ISCIII, 28031 Madrid, Spain

${ }^{9}$ Euromed Research Center, Euromed Faculty of Engineering, Euromed University of Fes (UEMF), Route de Meknès, 30000 Fès, Morocco

${ }^{10}$ Laboratoire de Chimie de Coordination du CNRS, 205 route de Narbonne,31077 Toulouse Cedex 4 France

${ }^{11}$ Université Toulouse 118 route de Narbonne, 31077 Toulouse Cedex 4, France

Corresponding authors: Mignani, S. (smignani@glycovax.com), Rodrigues, J. (joaor@uma.pt), Roy, R.

(rroy@glycovax.com), Majoral, J-P. (jean-pierre.majoral@lcc-toulouse.fr)

Keywords: dendrimer space; biocompatible dendrimers; dendrimers as drugs; physicochemical properties.

Teaser: Here, we highlight key in vitro physicochemical properties of biocompatible dendrimers necessary to increase their therapeutic use for medical applications are provided

Dendrimers are highly branched, star-shaped macromolecules with nanometer-scale dimensions that can be readily modified with a range of functional groups, thus modifying their physicochemical and biological properties. In nanomedicine, dendrimers can be used as vectors for the targeted delivery strategy of a variety of biologically active agents or can be used as drug per se. In the future, it will be necessary to designate and develop 'safe' dendrimers, which is currently a crucial concern. Here, we analyze the key in vitro physicochemical parameters to be considered for preclinical evaluation of biomedical dendrimers.

\section{Introduction}

Nanotechnology is an umbrella term referring to a multidisciplinary field that covers diverse sciences encompassing biology, chemistry, physics, and engineering based on nanomaterials with sizes ranging from the nanometer to micrometer. It has grown from a pure scientific interest to a major industry, with both commodity and specialty nanomaterial exposure to global populations and ecosystems. Indeed, nanotechnology has received significant attention in recent years in several domains, including the construction industry [1].

To circumvent several drawbacks in the development of drugs, nanotechnology approaches have been developed for the treatment of, for instance, cancer and other chronic human diseases. Generally speaking, the use of nanoparticles (NPs) as nanocarriers improves the bioavailability, targeting, and controlled-release profiles of drugs, both alone and in combination.

Several diverse NP types have been investigated for use as nanocarriers of drugs in several therapeutic fields, but mainly in oncology, in the form of: liposomes, albumin-based particles, biodegradable polymer-drug composites, polyethylene glycol (PEG)-ylated proteins, polymeric micelles, polymer-drug conjugate-based particles, inorganic particles, dendrons, and dendrimers. These latter compounds are a family of nanosized macromolecules, characterized by a highly homostructural branched 3D architecture and compact spherical geometry in solution. Dendrimers represent globular macromolecules, with highly branched 3D architecture, the shape and size of which can be controlled precisely [2-5]. Dendrimers represent ideal delivery vehicles, and there are high hopes for their future applications in nanomedicine [6].

Dendrimers have generated a large number of research publications, which has grown exponentially from around 2500 in 2006 to $\sim 8300$ publications in 2019 , with $\sim 25,000$ citations highlighted based on using 'dendrimer' 
as a keyword. In addition, the number of patents, in different applications, has also grown exponentially from four (between 1984 and 1990) to 739 (between 2005 and 2013) [7].

To date, several preclinical and clinical successes have been reported from dendrimers. For example, Roy et al. developed glycopeptide dendrimers as anticancer vaccine candidates [8]. In 2012, Starpharma Holdings started two pivotal Phase III trials for the treatment of bacterial vaginosis with VivaGel ${ }^{\circledR}$ (SPL7013 Gel, astodrimer sodium). This active polyanionic G4-poly(L-lysine)-type dendron has 32 naphthalene disulfonate groups on its surface and has shown potent topical vaginal microbicide activity. Starpharma has now received US Food and Drug Administration (FDA) Phase III approval. Starpharma/AstraZeneca recently advanced from Phase I to Phase II trials with a poly(lysine)dendrimer-based nanocarrier encapsulating docetaxel (DEP® docetaxel) showing superior anticancer activity against several important solid cancer types, including breast, prostate, lung, and ovarian tumors. Importantly, patients treated with $\mathrm{DEP}{ }^{\circledR}$ docetaxel showed no neutropenia adverse effects or lifethreatening toxicity seen in patients treated with conventional docetaxel formulations $[9,10]$.

Here, we analyze specific structural dendrimer characteristics that are useful in developing nanomedicine.

\section{Specific description of structural dendrimer characteristics in nanomedicine}

A schematic of typical dendritic architecture for biomedical applications is illustrated in Figure 1, using polyamidoamine (PAMAM) dendrimers as examples. Four different structural components define dendrimers: (i) a central core; (ii) an interior dendritic structure (named branches) comprising regularly repeating branching units attached to the core, and defining generations $\mathrm{G}_{n}$, where $n$ is $0,0.5,1,1.5$, etc.; (iii) an external surface with the possibility to graft multiple-type functional groups distributed in 3D space; and (iv) void spaces capable of accommodating molecular cargo. The main dendrimers developed in nanomedicine are the PAMAM, PPI, PEHAM, carbosilane, and phosphorus families [11].

Numerous chemical combinations of these four components yield nanomaterials of different sizes, shapes, and internal cores that are ideal candidates for nanosciences, including biological applications. The structural properties of these dendrimers include controllable internal cavities (void spaces) bearing specific species for the physical encapsulation of guest drugs and an external periphery that contains multiple functional groups to facilitate specific targeting and recognition. These fine-tuning chemical modifications allow modulation of the composition, architecture, and cellular behaviors of dendrimers, key properties to improve their in vitro and in vivo characteristics, such as their biocompatibility from cells to tissues and their pharmacokinetic/pharmacodynamic (PK/PD) activities [12,13].

Taken together, dendrimers used in nanomedicine should be biocompatible, biodegradable, easily targetable, useful for therapeutic and imaging purposes, monodispersed, safe, with high therapeutic windows, nonimmunogenic, and with precisely controllable dimensions and architectures. Figure 2 shows the main advantages of NPs and dendrimers in nanomedicine. The targeting strategy of dendrimers falls within the thematic of 'precision medicine' that includes the fields of diagnostic (imaging and identification) and therapeutic applications, such as delivery of drugs to exact location, killing cancer cells, bacteria, viruses and so on, and repair of damaged tissues.

However, the main disadvantages of dendrimers are: (i) a lack of knowledge of the effects of dendrimers in biochemical pathways and processes in mammalian systems; (ii) a lack of knowledge of the toxicity and exposure pathways; (iii) cost of development; and (iv) implementation issues. The main advantages of dendrimers in nanomedicine as nanocarriers or as drugs per se are: (i) reduce the dose of drug required; (ii) modulation of PK/PD; (iii) increase treatment efficacy related to unsolved medical problems; (iv) control biodistribution of drug; (v) decrease drug adverse effects and reduce mortality and morbidity; (vi) increase drug delivery to the target location; (vii) easy to detect with fluorescent probes, rendering them sensitive diagnostic tools; (viii) improve patient compliance; (ix) reduce unmet medical needs; (x) allow multiple routes of administration [e.g., intravenous (iv), intraperitoneal (ip), ocular, transdermal, oral (po), intranasal, and pulmonary] [14]; and (xi) high drug-loading capacity (local concentration effect).

Recently, researchers suggested the term 'dendrimer space concept' as a new approach for use by medicinal chemists to find original drug-based dendrimers. In this concept, the dendrimer space defines a new 'druggable' cluster that is included in the vast volume of chemical space, taking inspiration from the concepts of 'drug-likeness' and 'druggability', which are fully integrated into the practical drug discovery process [15].

\section{In vitro cytotoxicity and hemolytic activities of dendrimers}

The permeability of cell membranes to various families of dendrimers has been studied extensively. In early studies, Tajarobi and colleagues determined the permeability of cationic G0-4 PAMAM dendrimers across MadinDarby Canine Kidney (MDCK) cell lines and human epithelial colorectal adenocarcinoma (Caco-2) cell monolayers. Caco-2 cells are the most popular cellular model for studies on the passage and transport of molecular entities [16]. This cell line expresses in culture most of the morphological and functional characteristics of small intestinal absorptive cells and is suitable for molecular-permeability screening studies [17]. The permeability of PAMAM

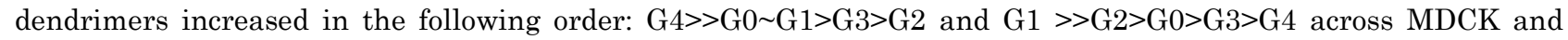
Caco-2 cells, respectively. G0-G2 dendrimers displayed an appreciable permeability that, combined with their low toxic effects on Caco-2 cells, suggests their potential use as water-soluble polymeric drug carriers for oral drug delivery [18]. In a separate study, the overall rank order of PAMAM dendrimer permeability was established as G3.5 $-\mathrm{CO}_{2} \mathrm{H}>\mathrm{G} 2-\mathrm{NH}_{2}>\mathrm{G} 2.5-\mathrm{CO}_{2} \mathrm{H}>\mathrm{G} 1.5-\mathrm{CO}_{2} \mathrm{H}>\mathrm{G} 2-\mathrm{OH}$. The strong permeability of cationic PAMAM dendrimers (except G2-NH2) was probably caused by the reversible reduction of trans-epithelial electrical resistance (TEER) 
and disruption of tight-junction proteins to open this channel [19]. In addition, surface $N$-acylation of PAMAM dendrimers decreased their cytotoxicity while maintaining the membrane permeability [20]. In addition, solid-state NMR studies showed that amphipathic dendrimer molecules can be stably incorporated into biomembranes [21]. In addition, improvement in permeability coefficients (Papp), through Caco-2 cell monolayers, of radiolabeled mannitol in the presence of simple dendrimers (G3-5-PAMAM dendrimers), which was more pronounced in the presence of lauryl conjugated dendrimers, was described by Jevprasesphant et al. [22].

Numerous studies have been carried out to evaluate the in vitro cytotoxicity of dendrimers using different cell lines, concentrations, incubation times, and assay methods. These studies have shown that cytotoxicity depends, to some extent, on the core structure, but is most strongly influenced by the nature of the functional groups on the dendrimer surface [23,24]. The cytotoxicity that induces necrosis of cells (rapid loss of membrane integrity) leading to decreased cell viability and the hemolytic toxicity, which is the breaking off of red blood cells leading to cell lysis and release of hemoglobin into plasma, is dependent on the generation, surface group functionality, and nature of the core and/or branches.

Several studies have highlighted the key role of surface functional groups for the control of cytotoxic properties. Cytotoxicity levels can be decreased by simple modifications of the terminal surface groups, such as PEGylation, acetylation, or the grafting of amino acid, peptide or carbohydrate units. [25]. An alternative strategy used to avoid toxicity is to prepare dendrimer scaffolds based on biocompatible units, such as those produced in known metabolic pathways [26-28]. For instance, high cytotoxicity has been observed for cationic PAMAM dendrimers displaying hydrophilic $\mathrm{NH}_{2}$ termini. In addition, cationic PAMAM dendrimers induced a significant decrease in cell viability against V79 Chinese hamster lung fibroblasts after incubation for $24 \mathrm{~h}$. Their cytotoxicity level depended on the generation number of the dendrimers: G3, $1 \mathrm{nM}$; G5, $10 \mathrm{nM}$; and G7, $100 \mathrm{nM}$ [29]. Further studies from several groups have also highlighted the key role of surface functional groups in the control of cytotoxic properties. Cytotoxicity levels can be decreased by simple modifications of the terminal surface groups. Nitrogen functions with decreased basicity display lower cytotoxicity, because of their decreased interactions with cell membranes. Thus, interaction and membrane bilayer hole creation studies using G7-PAMAM dendrimers against KB cancer cell membranes have been described. Large cationic dendrimers can disrupt cell membranes to facilitate the transport of drugs into cells [30-38]. Recently, Feliu et al. published a study of the toxicity of G1-G5 PAMAM dendrimers, with amino (cationic forms) or hydroxyl groups on their surface, for several cell lines, such as human cervical cancer (HeLa) and acute monocytic leukaemia cells (THP1), and monocyte-derived macrophages (HMDM) [39]. Excellent biocompatibility was observed for all dendrimers bearing hydroxyl groups, whereas the library of dendrimers with cationic groups exhibited dose-dependent and time-dependent cytotoxicity against HeLa, THP1 and HMDM cells, whereas neutral dendrimers did not.

Interestingly, the concept of dendrimer architectures and in vitro membrane bilayer hole formation was analyzed by the comparison of polycationic linear or dendritic polymers including PAMAM dendrimers, poly-Llysine, polyethyleneimine (PEI), diethylaminoethyl-dextran (DEAE-DEX), and neutral polymers as well as polyvinyl alcohol (PVA), and PEG against KB and Rat2 cell lines [40,41]. Membrane permeability was significantly enhanced by the most cationically charged polymer density, whereas the membrane permeability of PAMAM resulted from its spherical architecture, promoting its interactions with lipid membrane cells and then cell death. PVA and PEG showed no impact on membrane permeability. Similarly, G7-NH2 PAMAM dendrimers, but not G5NH2 or Ac-G5 dendrimers, were observed to form holes [42].

Figure 3 details the influence of cationic generation (G2-G4) PAMAM dendrimers on the in vitro viability of Caco-2 cells. The increase in cationic generation decreased cell viability, and for a considered generation, this viability decreased with the concentration of cationic PAMAM dendrimers. The same authors also reported the role of PAMAM dendrimer concentration and generation (G2, G4, and G6) on cell growth and cytotoxicity in HEK293T and HeLa cell lines [43].

Figure 4 details the in vitro viability of Caco-2 cells in response to $N$-acetylated G2-4 PAMAM dendrimers [20]. Cell viability decreased when the number of acetylated amino groups on the surface increased.

Hematological toxicity of dendrimers appears to be a major concern for dendrimers administered intravenously. Hemolytic activity, which is the rupture of red blood cells, can be studied in vitro by the evaluation of hemoglobin release across cell membranes, indicating potential cell membrane damage. Free cationic terminal groups of dendrimers interact with red blood cells leading to hemolysis. Hemolytic toxicity of dendrimers such as PPI, PAMAM and poly-L-lysine dendrimers has been investigated [28]. For instance, cationic PAMAM dendrimers showed generation-dependent hemolytic activity: G4 $>>$ G3 $>>$ G1 $>$ G4 . PAMAM dendrimers for the delivery of 5-FU showed hemolytic toxicity ( 15-17\%) [44]. G3 PAMAM and G3 PPI dendrimers displayed hemolytic effects above a concentration of $1 \mathrm{mg} / \mathrm{ml}$ [45], whereas, in a separate study, G4 and G5 PPI dendrimers showed hemolytic toxicity of $35.7 \%$ and $49.2 \%$, respectively [46].

G4-poly-L-lysine dendrimers induced hemolytic toxicity ( 14\%) [47]. However, no generation-dependent activity was observed for cationic PEI-based diaminobutane (DAB) dendrimers. In general, higher-generation cationic dendrimers induced greater hemolytic toxicity, which might be attributed to their greater overall cationic charge. By contrast, anionic PAMAM dendrimers and PEGylated melamine dendrimers did not display any hemolytic activity. In addition, no morphological cell changes were observed for half-anionic G3.5-9.5 PAMAM dendrimers ( $\mathrm{CO}_{2} \mathrm{H}$-surface groups) [48] (reviewed in [49]). 
The most common strategies for the development of dendrimers with lower cytotoxicity are the following: PEGylation; use of carbohydrate engineered dendrimers; acylation; use of anionic dendrimers; and grafting of amino acids, such as phenylalanine and glycine or peptides [25,50,51].

An alternative strategy to avoid toxicity is to prepare dendrimer scaffolds based on biocompatible units, such as those produced in known metabolic pathways. To date, the main biocompatible dendrimeric scaffolds are: polyether dendrimers [52,53]; bow-tie dendrimers based on PEG and bis-hydroxymethyl-propionic acid [26]; polyester dendrimers [27]; PEI dendrimers [54], polyether-copolymer dendrimers [55], phosphate dendrimers [56], melamine dendrimers [57], 3,4-dihydroxy-L-phenylalanine (L-DOPA) dendrimers [58], peptide dendrimers [59], phosphorusbased dendrimers [60], sugar-based scaffolds [61], and polyurea dendrimers [62]. A nontoxic polypropylimine dendrimer, called PETIM, has been also described [28].

Figure 2 presents representative symmetric and asymmetric biodegradable/biocompatible core/branches of dendrimers. For example, polyether and polyester dendrimer-poly(ethylene oxide) bow-tie hybrids were developed by Fréchet et al. [4,63], whereas phosphorus dendrimers were developed by Majoral and Caminade [64-66]. In recent years, several groups simultaneously developed an original strategy, the self-immolative dendrimer strategy, involving the dissociation of dendrimers for molecular amplification as dendritic prodrug systems [67]. The starting molecule was equipped with a focal trigger, which, once activated by a single activation, initiated the fragmentation process (i.e., complete breakdown of the linker) inducing the spontaneous release of all the end-group molecules, which can be biologically active compounds, in a domino-like manner. Figure 5 provides a schematic representation of the cytotoxicity effects related to the generation [36], surface characteristics [25,37,68], nature of core and branches [38], and concentration of dendrimers used. The influence of the generation and the nature of the surface of dendrimers can be demonstrated as follows: the in vitro cytotoxicity against Caco-2 cells of polycationic dendrimers increased with dendrimer generation (e.g., PAMAM), whereas polyanionic dendrimers showed no or low cytotoxicities regardless of the generation. Cationic dendrimers (e.g., PAMAM) grafted with arginine, lysine, or amidine groups showed higher in vitro cytotoxicity against, for example, 293 human kidney cells. Anionic dendrimers [e.g., PAMAM, diaminobutane dendrimers (DAB)] showed low cytotoxicity against cell. Dendrimers such as PAMAM, PPI, or carbosilane dendrimers with poly(ethylene oxide) (PEO) groups grafted onto their surfaces, resulting in, for example, Csi-PEO dendrimers, or with the introduction of hydroxyl terminated PEO groups on their surface, displayed low in vitro cytotoxicity against cells. The influence of the core/branches on the in vitro cytotoxicity against cells follows the order: $\mathrm{PETIM}<\mathrm{PPI}<\mathrm{PAMAM}$ and $\mathrm{DAB}<\mathrm{PEI}$.

\section{Concluding remarks and perspectives}

Taken together, physicochemical properties, such as charge, surface properties, shape, and size, of NPs in general and dendrimers in particular, as well as the route of administration, strongly influence PK by control ling absorption, distribution, and elimination processes. The final goal is the improvement of the plasma residence time and half-life of dendrimers, whether as nanocarriers or not (e.g., as drugs), as well as their tissue permeation, delivering the drug to the target tissue and avoiding adverse effects. Most dendrimers are used as nanocarriers of small and large molecules, genes and peptides. to: (i) enhance the therapeutic potency of drugs by improving their stability under physiological conditions, improving aqueous solubility, improving PK/PD behaviors, preventing recognition by macrophages, and elimination by the reticuloendothelial system, increasing their circulation half-life and selective passive diffusion via the enhanced permeability and retention effect [69], and overcoming low oral bioavailability; (ii) carry out targeted drug delivery; and (iii) allow nonclassical routes of administration.

It is clear that 'safe', nontoxic dendrimers will be mandatory for their clinical developments as both drugdelivery systems and nanodrugs. The two main strategies to avoid dendrimer toxicity are based on the design and synthesis of a biodegradable/biocompatible core and branching units, masking peripheral charges by using surface engineering approaches.

For go/no-go decision-making by project teams earlier during the development process, a list of the desired basic requirements for a dendrimer to become a clinical candidate and to move towards investigational new drug (IND) submission has recently been published [70]. To this end, here, we have outlined and analyzed fully the key parameters to be considered to secure the development of dendrimers for testing in clinical trials and to enhance their translation into the clinic.

\section{Acknowledgments}

S.M. and J.R. acknowledge the support of Fundacão para a Ciência e a Tecnologia with Portuguese Government funds through the CQM Strategic Project PEstOE/QUI/UI0674/2013, and Agência Regional para o Desenvolvimento da Investigação Tecnologia through the project M1420-01-0145-FEDER-000005 - Centro de Química da Madeira - CQM1 (Madeira 14-20 Program). V.C acknowledges the support of the Spanish Ministerio de Economía y Competitividad (grant no. SAF201789288-R from MINECO/AEI/FEDER/UE). J-P.M. thanks the CNRS (France) for financial support. The authors would like to thank Helena Tomas and Maria Zablocka for providing valuable feedback.

\section{References}

1 Silvestre, J. et al. (2016) Review on concrete nanotechnology. Eur. J. Environ. Civ. Eng. 20, 455-485

2 Abbasi, E. et al. (2014) Dendrimers: Synthesis, applications, and properties. Nanoscale Res. Lett. 9, 1-10

3 Augustus E. N. et al. (2017) A review of synthesis, characterization and applications of functionalized dendrimers. Am. J. Polym. Sci. 7, 814

4 Grayson, S.M. and Fréchet, J.M.J. (2001) Convergent dendrons and dendrimers: from synthesis to applications. Chem. Rev. 101, 3819-3867

5 Azzouz, A. and Roy, R. (2017) Dendrimers: syntheses, toxicity, and applications toward catalysis, environmental sciences, and nanomedecine. Can. J. Chem. 95, v-vii

6 Felder-Flesch, D. (2016) Dendrimers in Nanomedicine, Pan Stanford Publishing

7 Kalhapure, R. S. et al. (2015) Dendrimers - from organic synthesis to pharmaceutical applications: an update. Pharm. Dev. Technol. 20, 2240

8 Roy, R. (2004) New trends in carbohydrate-based vaccines. Drug Discovery' Today Technol. 1, 327-336 
9 Rupp, R. et al. (2007) VivaGelTM (SPL7013 Gel): a candidate dendrimer - microbicide for the prevention of HIV and HSV infection. Int. J. Nanomedicine 2, 561-566

10 Starpharma. https://starpharma.com/ [Accessed 26 February 2019]

11 Caminade, A.M. and Majoral, J.P. (2018) Which dendrimer to attain the desired properties? Focus on phosphohydrazone dendrimers. Molecules 23, E622

12 Wijagkanalan, W. et al. (2011) Designing dendrimers for drug delivery and imaging: pharmacokinetic considerations. Pharm. Res. 28, 15001519

13 Svenson, S. and Tomalia, D. A. (2005) Dendrimers in biomedical applications - reflections on the field. Adv. Drug Deliv. Rev. 57, 2106-2129

14 Mignani, S. et al. (2013) Expand classical drug administration ways by emerging routes using dendrimer drug delivery systems: a concise overview. Adv. Drug Deliv. Rev. 65, 1316-1330

15 Mignani, S. et al. (2013) Dendrimer space concept for innovative nanomedicine: a futuristic vision for medicinal chemistry. Prog. Polym. Sci. 38, 993-1008

16 Tajarobi, F. et al. (2001) Transport of poly amidoamine dendrimers across Madin-Darby canine kidney cells. Int. J. Pharm. 215, 263-267

17 Le Ferrec, E. et al. (2001) In-vitro models of the intestinal barrier: the report and recommendations of ECVAM workshop 46. Altern. Lab. Anim. 29, 649-668

18 El-Sayed, M. et al. (2002) Transepithelial transport of poly(amidoamine) dendrimers across Caco-2 cell monolayers. J. Control. Release 81, $355-365$

19 Kitchens, K.M. et al. (2006) Transport of poly(amidoamine) dendrimers across Caco-2 cell monolayers: Influence of size, charge and fluorescent labeling. Pharm. Res. 23, 2818-2826

20 Kolhatkar, R.B. et al. (2007) Surface acetylation of polyamidoamine (PAMAM) dendrimers decreases cytotoxicity while maintaining membrane permeability. Bioconjug. Chem. 18, 2054-2060

21 Smith, P.E.S. et al. (2010) Solid-state NMR reveals the hydrophobic-core location of poly(amidoamine) dendrimers in biomembranes. J. Am. Chem. Soc. 132, 8087-8097

22 Jevprasesphant, R. et al. (2003) Engineering of dendrimer surfaces to enhance transepithelial transport and reduce cytotoxicity. Pharm. Res. 20, 1543-1550

23 Mukherjee, S. P. et al. (2010) In-vitro mammalian cytotoxicological study of PAMAM dendrimers - towards quantitative structure activity relationships. Toxicol. Vitr. 24, 169-177

24 Duncan, R. and Gaspar, R. (2011) Nanomedicine(s) under the microscope. Mol. Pharm. 8, 2101-2141

25 Mishra, V. et al. (2009) Surface-engineered dendrimers: a solution for toxicity issues. J. Biomater. Sci. Polym. Ed. 20, 141-166

26 Lee, C.C. et al. (2006) A single dose of doxorubicin-functionalized bow-tie dendrimer cures mice bearing C-26 colon carcinomas. Proc. Natl. Acad. Sci. U. S. A. 103, 16649-16654

27 Ihre, H.R. et al. (2002) Polyester dendritic systems for drug delivery applications: design, synthesis, and characterization. Bioconjug. Chem. $13,443-452$

28 Jain, S. et al. (2010) Poly propyl ether imine (PETIM) dendrimer: a novel non-toxic dendrimer for sustained drug delivery. Eur. J. Med. Chem. 45, 4997-5005

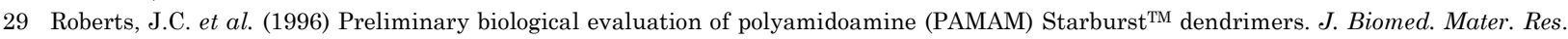
$30,53-65$

30 Haensler, J. and Szoka, F. C. (1993) Polyamidoamine cascade polymers mediate efficient transfection of cells in culture. Bioconjug. Chem. 4, $372-379$

31 Fischer, D. et al. (1999) A novel non-viral vector for DNA delivery based on low molecular weight, branched polyethylenimine: effect of molecular weight on transfection efficiency and cytotoxicity. Pharm. Res. 16, 1273-1279

32 Malik, N. et al. (1999) Dendrimer-platinate: a novel approach to cancer chemotherapy. Anticancer Drugs 10, 767-776

33 Chen, H-T. et al. (2004) Cytotoxicity, hemolysis, and acute in vivo toxicity of dendrimers based on melamine, candidate vehicles for drug delivery. J. Am. Chem. Soc. 126, 10044-10048

34 Yang, H. et al. (2007) Synthesis and characterization of nanoscale dendritic RGD clusters for potential applications in tissue engineering and drug delivery. Int. J. Nanomedicine 2, 89-99

35 Tomalia, D.A. et al. (2007) Dendrimers as multi-purpose nanodevices for oncology drug delivery and diagnostic imaging. Biochem. Soc. Trans. 35, 61-67

36 Jevprasesphant, R. et al. (2003) The influence of surface modification on the cytotoxicity of PAMAM dendrimers. Int. J. Pharm. 252, 263266

37 Naha, P.C. et al. (2018) Toxicology of engineered nanoparticles: focus on poly(amidoamine) dendrimers. Int. J. Environ. Res. Public Health $15,338-356$

38 Neerman, M.F. et al. (2004) Reduction of drug toxicity using dendrimers based on melamine. Mol. Pharm. 1, 390-393

39. Feliu, N. et al. (2012) Stability and biocompatibility of a library of polyester dendrimers in comparison to polyamidoamine dendrimers. Biomaterials 33, 1970-1981

40 Hong, S. et al. (2007) The binding avidity of a nanoparticle-based multivalent targeted drug delivery platform. Chem. Biol. 14, 107-115

41 Wolinsky, J.B. and Grinstaff, M.W. (2008) Therapeutic and diagnostic applications of dendrimers for cancer treatment. Adv. Drug Deliv. Rev. 60, 1037-1055

42 Hong, S. et al. (2004) Interaction of poly(amidoamine) dendrimers with supported lipid bilayers and cells: hole formation and the relation to transport. Bioconjug. Chem. 15, 774-782

43 Parimi, S. et al. (2010) Mechanistic insight into cell growth, internalization, and cytotoxicity of PAMAM dendrimers. Biomacromolecules 11, 382-389

44 Bhadra, D. et al. (2003) A PEGylated dendritic nanoparticulate carrier of fluorouracil. Int. J. Pharm. 257, 111-124

45 McNerny, D.Q. et al. (2010) Understanding specific and nonspecific toxicities: a requirement for the development of dendrimer-based pharmaceuticals. Wiley Interdiscip. Rev. Nanomedicine Nanobiotechnol. 2, 249-259

46 Bhadra, D. et al. (2005) Glycodendrimeric nanoparticulate carriers of primaquine phosphate for liver targeting. Int. J. Pharm. 295, 221-233

47 Agrawal, P. et al. (2007) Glycoconjugated peptide dendrimers-based nanoparticulate system for the delivery of chloroquine phosphate. Biomaterials 28, 3349-3359

48 Jain, K. et al. (2010) Dendrimer toxicity: let's meet the challenge. Int. J. Pharm. 394, 122-142

49 Labieniec, M. and Watala, C. (2009) PAMAM dendrimers - diverse biomedical applications. Facts and unresolved questions. Cent. Eur. J. Biol. 4, 434-451

50 Mintzer, M.A. and Grinstaff, M.W. (2011) Biomedical applications of dendrimers: a tutorial. Chem. Soc. Rev. 40, 173-190

51 Duncan, R. and Izzo, L. (2005) Dendrimer biocompatibility and toxicity. Adv. Drug Deliv. Rev. 57, 2215-2237

52 Appelhans, D. et al. (2000) Synthesis and characterization of poly(ether amide) dendrimers containing different core molecules. Macromolecules 33, 9494-9503

53 Hawker, C.J. and Fréchet, J.M.J. (1990) Preparation of polymers with controlled molecular architecture. A new convergent approach to dendritic macromolecules. J. Am. Chem. Soc. 112, 7638-7647

54 Krishna, T.R. et al. (2006) Water-soluble dendrimeric two-photon tracers for in vivo imaging. Angew. Chem. Int. Ed. 45, 4645-4648 
55 Dhanikula, R.S. and Hildgen, P. (2007) Influence of molecular architecture of polyether-co-polyester dendrimers on the encapsulation and release of methotrexate. Biomaterials 28, 3140-3152

56 Domański, D.M. et al. (2004) Preliminary evaluation of the behavior of fifth-generation thiophosphate dendrimer in biological systems. Biomacromolecules 5, 2007-2012

57 Simanek, E.E. et al. (2010) The 8 year thicket of triazine dendrimers: strategies, targets and applications. Proc. R. Soc. A Math. Phys. Eng. Sci. 466, 1445-1468

58 Tang, S. et al. (2006) Synthesis and characterization of water-soluble and photostable L-DOPA dendrimers. Org. Lett. 8, 4421-4424

59 Gu, F.X. et al. (2007) Targeted nanoparticles for cancer therapy. Nano Today 2, 14-21

60 Poupot, M. et al. (2006) Design of phosphorylated dendritic architectures to promote human monocyte activation. FASEB J. 20, 2339-2351

61 Roy, R. et al. (2015) These glyconanosynthons as powerful scaffolds and building blocks for the rapid construction of multifaceted, dense and chiral dendrimers. Chem. Soc. Rev. 44, 3924-3941

62 Restani, R.B. et al. (2012) Biocompatible polyurea dendrimers with pH-dependent fluorescence. Angew. Chem. Int. Ed., 51, $5162-5165$

63 Van Der Poll, D.G. et al. (2010) Design, synthesis, and biological evaluation of a robust, biodegradable dendrimer. Bioconjug. Chem. 21, $764-773$

64 Katir, N. et al, (2015) Synthesis of onion-peel nanodendritic structures. Chem. Eur. J. 17, 6400-6408

65 Maraval, V. et al. (2003) Lego chemistry for the straightforward synthesis of dendrimers. J. Org. Chem. 68, 6043-6046

66 Caminade, A.M. et al. (2012) Janus dendrimers synthesis and properties, New J. Chem. 36, 217-226

67 Roth, M.E. et al. (2016) Dendritic, oligomeric, and polymeric self-immolative molecular amplification. Chem. Rev. 116, 1309-1352

68 Luong, D. et al. (2016) PEGylated PAMAM dendrimers: enhancing efficacy and mitigating toxicity for effective anticancer drug and gene delivery. Acta Biomater. 43, 14-29

69 Nakamura, H et al. (2015) Development of next-generation macromolecular drugs based on the EPR effect: challenges and pitfalls. Expert Opin. XXXX 12, 53-64

70 Mignani, S. et al. (2017) Bench-to-bedside translation of dendrimers: reality or utopia? A concise analysis. Adv. Drug Deliv. Rev. XX, YYYZZZ

Figure 1. Schematic 2D representation of a dendrimer using polyamidoamine (PAMAM) dendrimers as a model with core, void spaces, interior branching, and surface group elements indicated.

Figure 2. Schematic of representative symmetric and asymmetric biodegradable/biocompatible core/branches of dendrimers: (a) polyether dendrimers [4,63], (b) polyester-dendrimers-poly(ethylene oxide) bow-tie hybrids [4,63], (c) azabis(dimethyl)phosphate-based dendrimers [64-66], and (d) self-immolative dendrimers $[4,63, X X, Y Y]$.

Figure 3. Influence of cationic (G2-G4) polyamidoamine (PAMAM) dendrimers on in vitro Caco-2 cell viability. The increase in cationic generation decreased cell viability (a) and, for a considered generation (b), this viability decreased with the concentration of cationic PAMAM dendrimers.

Figure 4. In vitro viability of Caco-2 cells with $\mathrm{N}$-acetylated G2-4 polyamidoamine (PAMAM) dendrimers. The cell viability decreased when the number of acetylated amino groups on the surface increased.

Figure 5. Schematic representation of the in vitro cytotoxic effects of dendrimers based on generation, surface characteristics, core, branches, and concentration of dendrimers. The in vitro cytotoxicity of polycationic dendrimers increases with their generation, whereas polyanionic or neutral dendrimers show no/low cytotoxicity regardless of the generation. The influence of the core/branches on the in vitro cytotoxicity against cells follows the order: PETIM $<P P I<$ polyamidoamine (PAMAM) and polyethyleneimine (PEI)-based diaminobutane (DAB)<PEI. 


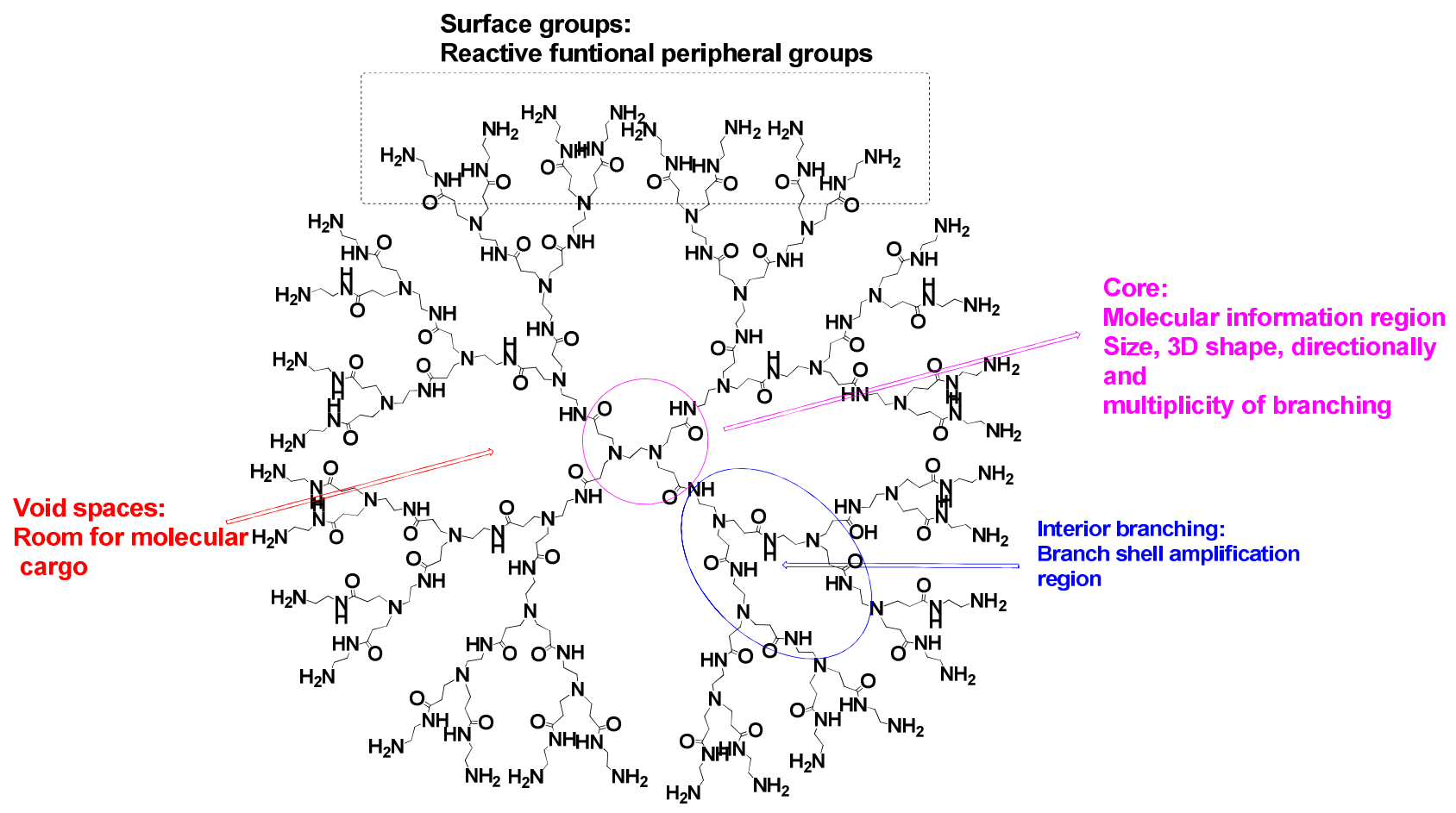




\section{Symmetric dendrimer}

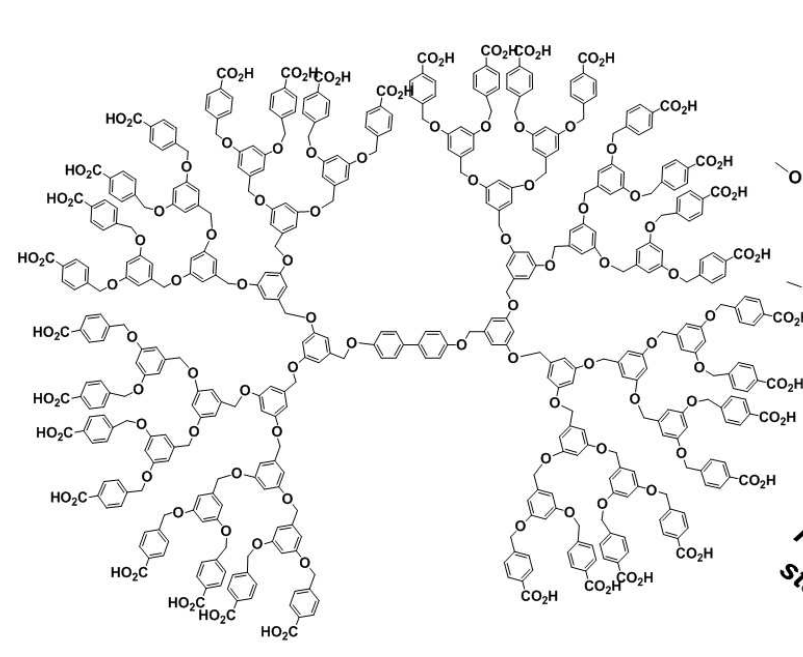

Polyether dendrimers (Frechet et al.)

\section{Symmetric dendrimer}

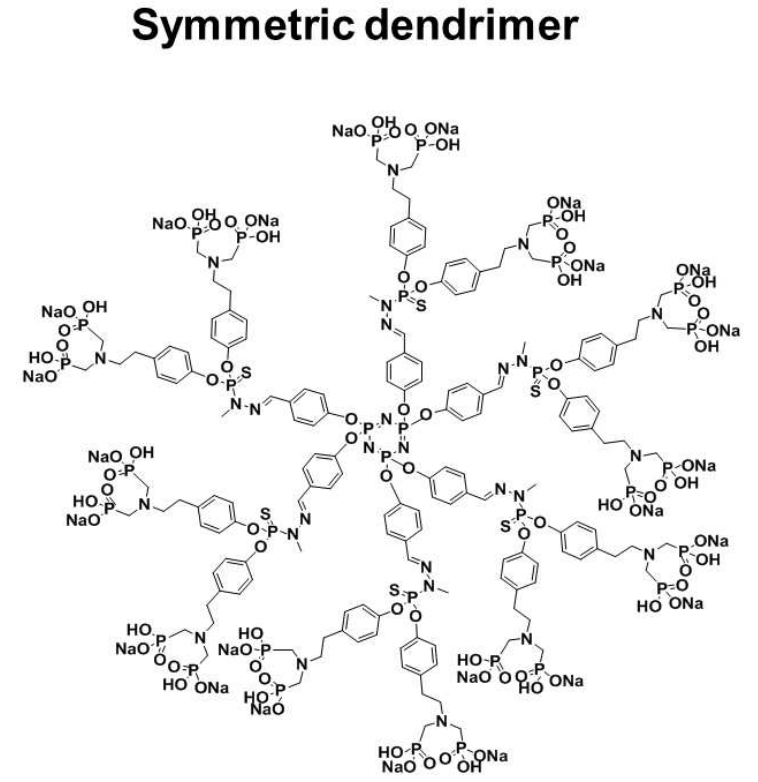

Azabis(dimethyl)phosphonate-based dendrimer (Caminade and Majoral et al.)
Asymmetric dendrimer

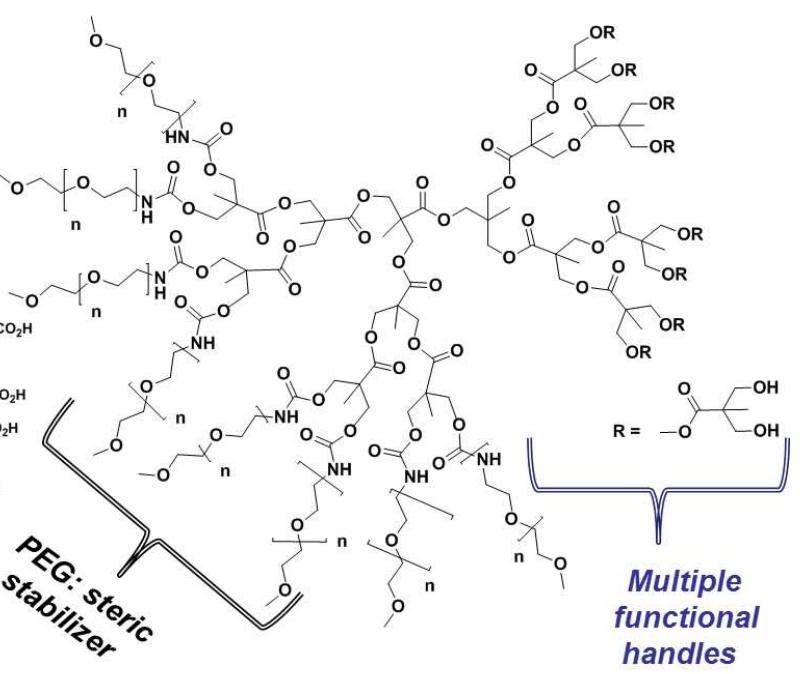

Polyester dendrimer-poly(ethylene oxide) bow-tie hybrids (Fréchet et al.)

\section{Geometrically disassembling process}

Linear diassembly Geometric diassembly

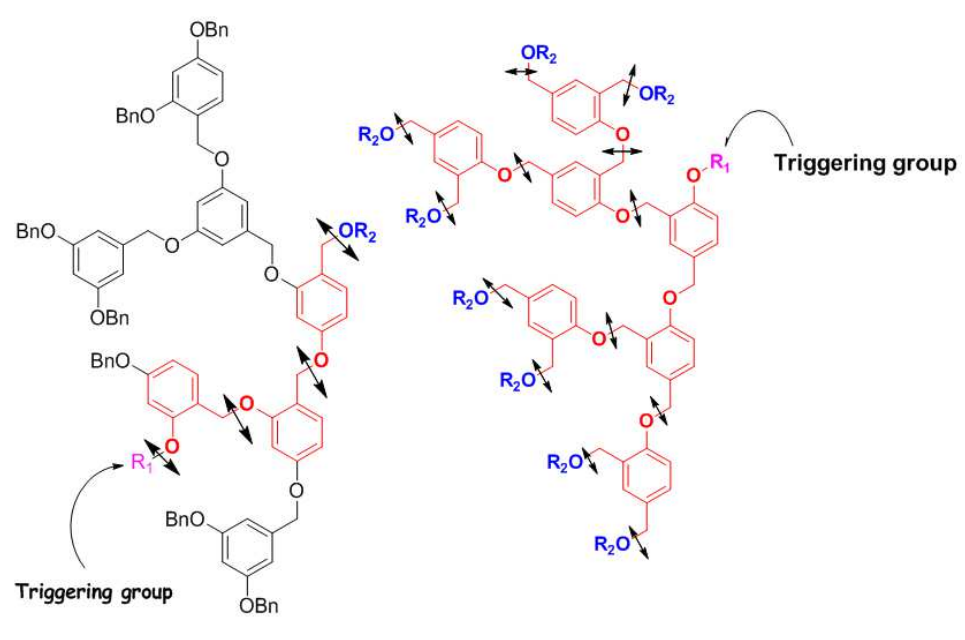

Self-immolative dendrimers

(Fréchet et al., Shabat et al., Thonthwaite et al.) 
(a)

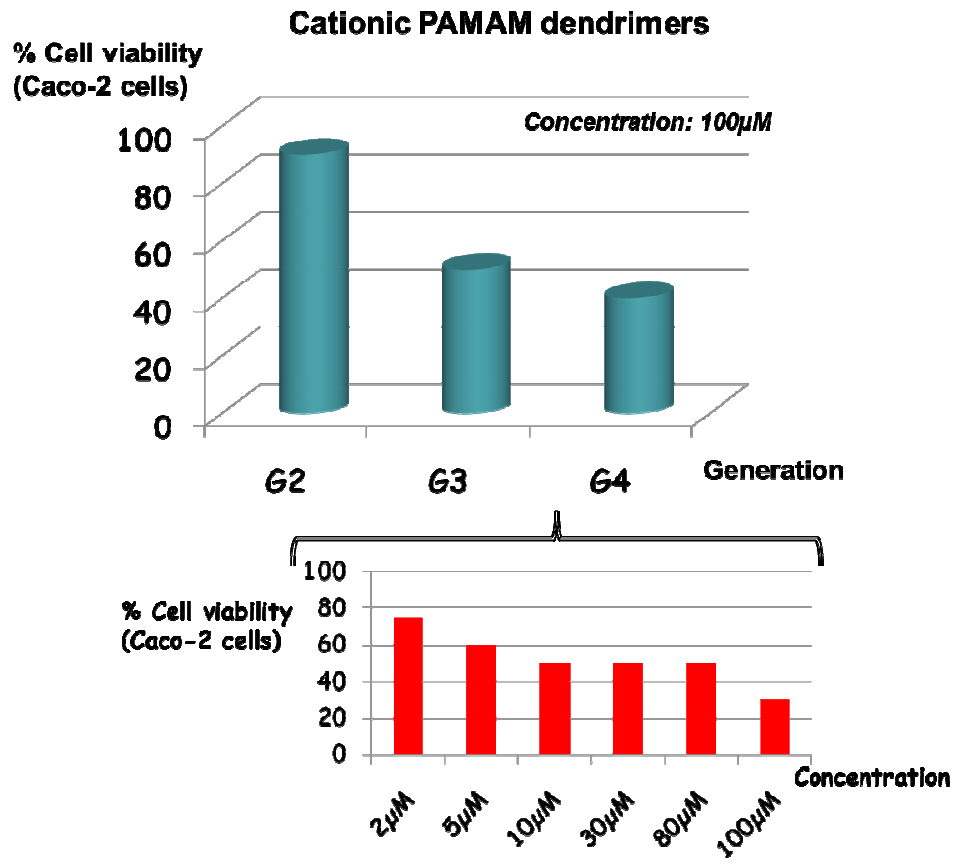



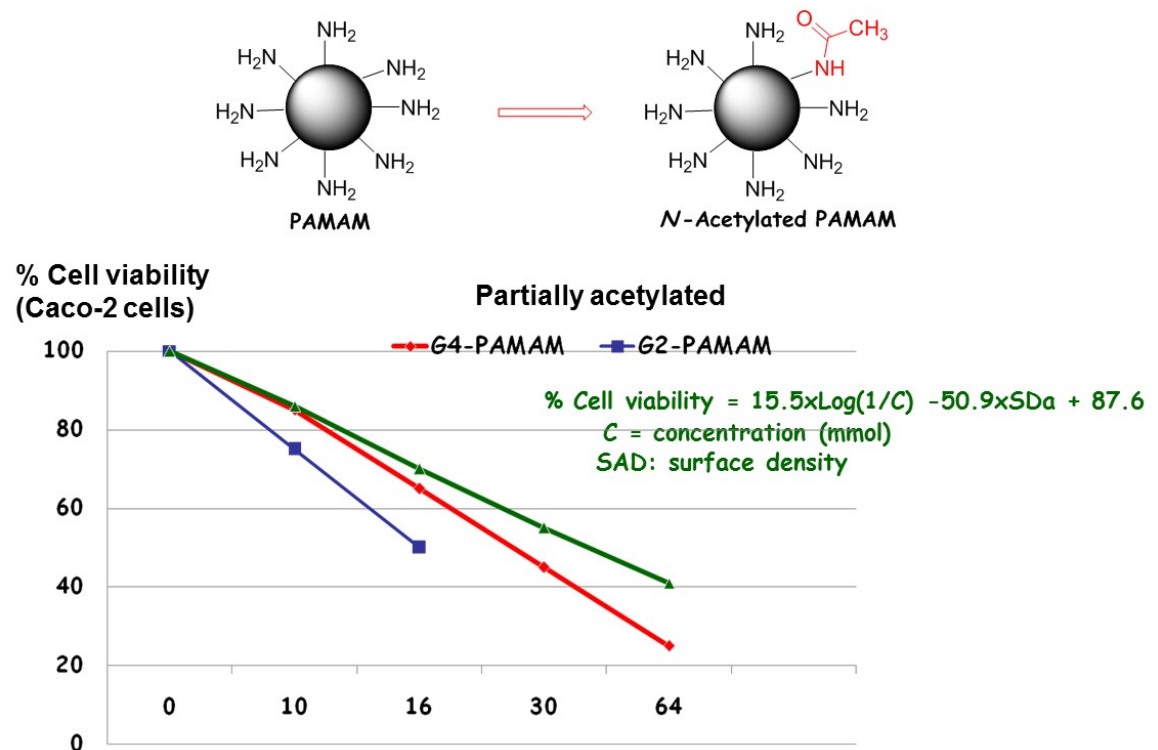

Number of unprotected amino groups or surface density (SAD, number of amines $/ \mathrm{nm}^{2}$ ) $\times 100$ 


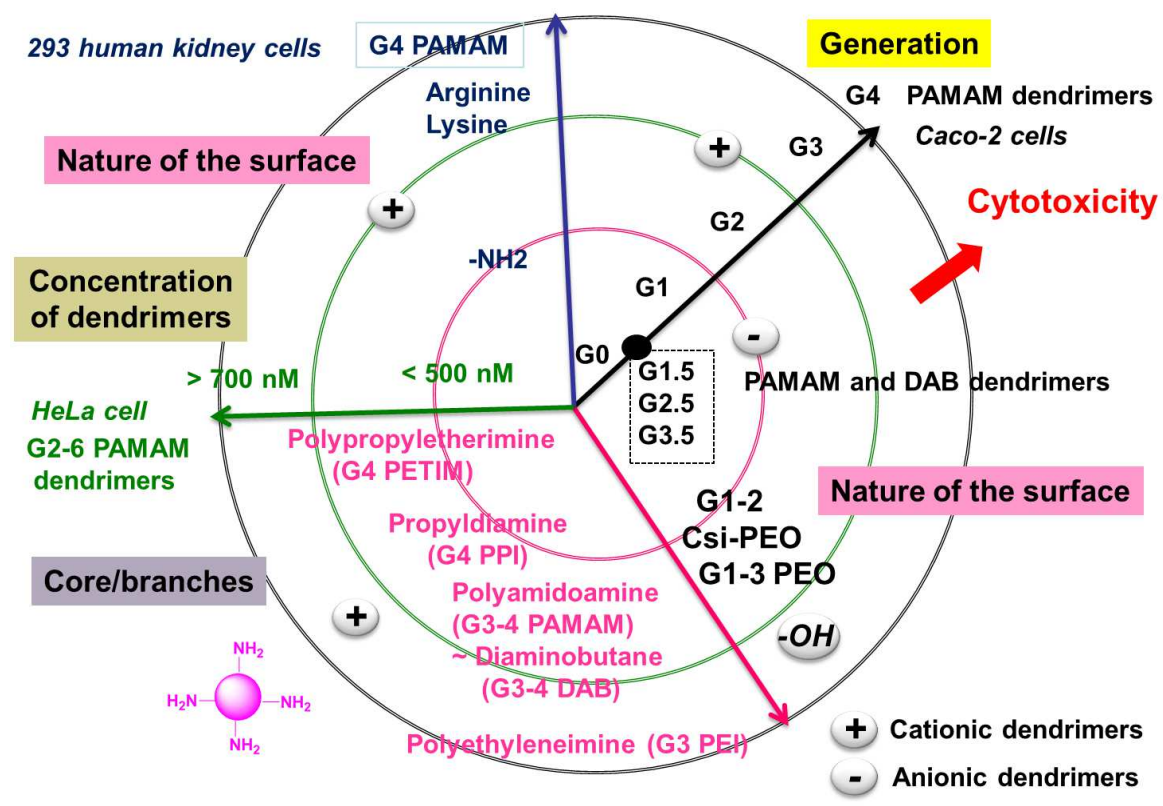




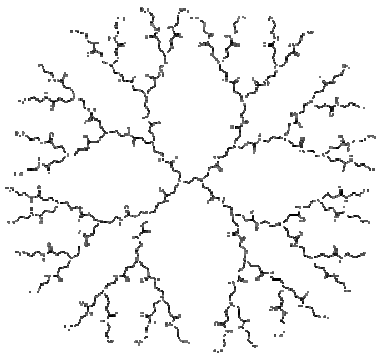

in vitro physicochemical

parameters analysis

Nanomedicine applications

Biomedical dendrimers 